\title{
A General Algorithm for Nonnegative Quadrature Formulas*
}

\author{
By M. Wayne Wilson
}

Abstract. A general algorithm is presented for determining numerical integration formulas exact for an arbitrary finite set of continuous functions defined on a compact set, involving nonnegative combinations of function values at a finite number of points in the set. Examples are given.

1. Introduction. The purpose of this paper is to present an algorithm for obtaining one- and multi-dimensional quadrature formulas of the form

$$
\int_{T} \omega(t) f(t) d t=\sum_{i=0}^{n} \lambda_{i} f\left(t_{i}\right), \quad \omega(t) \geqq 0
$$

where the base points $t_{0}, t_{1}, \cdots, t_{n}$ are within the compact region $T$, the coefficients $\lambda_{0}, \lambda_{1}, \cdots, \lambda_{n}$ are nonnegative, and where the formula is exact for the span of $n+1$ given functions defined on $T$. The advantages of formulas satisfying these properties is well known in the lore of numerical analysis, and will not be discussed here.

Let $T$ be a compact set of $E^{r}$. Let $C_{n}(T)$ be the span of $n+1$ continuous linearly independent functions $\phi_{0}(t), \cdots, \phi_{n}(t)$, defined on $T$. ( $T$ necessarily contains at least $n+1$ points.) A nonnegative linear functional is a linear functional $L$ such that $L(p) \geqq 0$ whenever $p(t) \geqq 0$ on $T$. Such an example is the integral,

$$
L(f) \equiv \int_{T} \omega(t) f(t) d T, \quad \omega(t) \geqq 0 .
$$

Except in degenerate situations, this is actually strictly positive, i.e. $p(t) \geqq 0$, $p(t) \not \equiv 0, \Rightarrow L(p)>0$.

Now, it is known that if $C_{n}(T)$ satisfies the Kreĭn condition $\left(\exists p(t) \in C_{n}(T)\right.$, s.t. $p(t) \geqq \alpha>0$ on $T$ ), and if $L$ is a nonnegative linear functional, then there exist points $t_{0}, t_{1}, \cdots, t_{n} \in T$, and nonnegative scalars $\lambda_{0}, \lambda_{1}, \cdots, \lambda_{n}$, such that

$$
L(f)=\sum_{i=0}^{n} \lambda_{i} f\left(t_{i}\right), \quad \forall f \in C_{n}(T) .
$$

Various proofs are given in Rogosinski [4], Tchakaloff [6], Wilson [7], [8], and Davis [1], [2]. (The KreIn condition is no real restriction, since, if it does not hold, we can append $\phi_{n+1} \equiv 1$ to the set $\phi_{0}, \phi_{1}, \cdots, \phi_{n}$, and work with the new set. A quadrature formula, exact for the new set, is exact for the given set, at the cost of at most one extra base point or function evaluation.) The proofs of Davis are constructive and provide a theoretical basis for the algorithm given in Section 2 . He showed that if $\left\{P_{i}\right\}$ is a dense sequence in $T$, and $L$ is a strictly positive linear functional, then for $N$ sufficiently large, there exist constants $\lambda_{0}, \lambda_{1}, \cdots, \lambda_{N}$, nonnegative, such that

Received August 14, 1968; revised October 9, 1968.

* This work was supported by the Office of Naval Research under Contract Nonr-562(36) at Brown University. 


$$
L(f)=\sum_{i=0}^{N} \lambda_{i} f\left(P_{i}\right), \quad \forall f \in C_{n}(T) .
$$

If $T_{N}=\left\{t_{0}, t_{1}, \cdots, t_{N}\right\}$ is a finite subset of $T$, we say that $T_{N}$ supports a nonnegative quadrature formula w.r.t. $\phi_{0}, \phi_{1}, \cdots, \phi_{n}$ if there exist nonnegative constants $\lambda_{0}, \lambda_{1}, \cdots, \lambda_{N}$ such that

$$
L(f)=\sum_{i=0}^{N} \lambda_{n} f\left(t_{i}\right), \quad \forall f \in C_{n}(T) .
$$

Davis' proof then shows how to construct a set $T_{N}$ which supports a nonnegative quadrature formula. Once we obtain the expression (1.2) (where $N \geqq n$, usually) we may use the Steinitz Algorithm to reduce the expression to the form (1.1). To use this algorithm, note that (1.2) is equivalent to the system of equations

$$
\mathbf{c}=\sum_{i=0}^{N} \lambda_{i} \phi\left(t_{i}\right)
$$

where $\mathbf{c}=\left(L \phi_{0}, L \phi_{1}, \cdots, L \phi_{n}\right)^{t}, \boldsymbol{\phi}(t)=\left(\phi_{0}(t), \phi_{1}(t), \cdots, \phi_{n}(t)\right)^{t}$.

We now outline the Steinitz Algorithm. Let $\mathbf{c}=\sum_{i=0}^{N} \lambda_{i} \mathbf{x}_{i}$, where $\lambda_{i}>0$, and assume $\mathbf{x}_{0}, \mathbf{x}_{1}, \cdots, \mathbf{x}_{N}$ are linearly dependent. Thus, an expression

$$
\mathbf{0}=\sum_{i=0}^{N} \alpha_{i} \mathbf{x}_{i}
$$

exists, where some $\alpha_{i}>0$. Define

$$
\sigma=\max \left\{\alpha_{i} / \lambda_{i}, i=0,1, \cdots, N\right\}>0 .
$$

Then

$$
\mathbf{c}=\frac{1}{\sigma}(\sigma \mathrm{c}-0)=\sum_{i=0}^{N}\left(\frac{\sigma \lambda_{i}-\alpha_{i}}{\sigma}\right) \mathbf{x}_{i}
$$

and, not only are all the coefficients still nonnegative, at least one vanishes. Thus by repeating this process, we can reduce the strictly positive expression $\mathrm{c}=\sum_{i=0}^{N} \lambda_{i} \mathbf{x}_{i}$ to a strictly positive combination of linearly independent vectors.

Immediately, if $T$ can be decomposed into subregions where known rules exist, we can formulate a rule like (1.2), and thus derive a rule like (1.1). A simple example of this would be integration of polynomials over a sum of disjoint intervals. Cross product regions can be treated similarly. (See Davis [1].)

2. Algorithm. If we suppose that a rule (1.2) exists, then we have a solution to the system of (nonlinear) simultaneous equation, $N \geqq n$,

$$
\sum_{j=0}^{N} \lambda_{j} \phi_{i}\left(t_{j}\right)=c_{i}, \quad i=0,1,2, \cdots, n,
$$

where $c_{i} \equiv L \phi_{i}, \lambda_{i} \geqq 0$, or, in matrix form

$$
\Phi(n, N) \lambda=\mathrm{c}
$$

where $\lambda$ is an $(N+1)$-vector, and $\mathbf{c}$ an $(n+1)$-vector. The unknowns are, of course, $t_{0}, t_{1}, \cdots, t_{N}$, and $\lambda_{0}, \lambda_{1}, \cdots, \lambda_{N}$. 
On the other hand, if we assume a set of $(N+1)$ points, $T_{N} \equiv\left\{t_{0}, t_{1}, \cdots, t_{N}\right\}$ $\subset T$, then the system is linear, and, using a standard linear programming algorithm like the simplex method, one can determine if the system

$$
\Phi(n, N) \boldsymbol{\lambda}=\mathrm{c}
$$

has a nonnegative solution, $\lambda^{*} \geqq 0$ (component-wise), by exhibiting one. (There will in general be an infinite number of nonnegative solutions, if there are any. However, if a L. P. algorithm determines a solution, it has at most $n+1$ nonnegative components, the rest being zero, and of course $T_{N}$ supports a nonnegative quadrature formula.)

Thus, our algorithm can now be stated:

Given: $\phi_{0}(t), \phi_{1}(t), \cdots, \phi_{n}(t)$.

Given: $L$, strictly positive linear functional.

Given: $\mathrm{c}=\left(L \phi_{0}, L \phi_{1}, \cdots, L \phi_{n}\right)^{t}$.

Given: An infinite sequence of point sets

$$
T_{N}=\left\{t_{0}^{(N)}, t_{1}^{(N)}, \cdots, t_{N}^{(N)}\right\}, \quad N=N_{1}, N_{2}, N_{3}, \cdots,
$$

where $n<N_{1}<N_{2}<N_{3}$

1. Set $i=1$.

2. Set $N=N_{i}$.

3. Calculate the matrix $\Phi(n, N)=\phi_{i}\left(t_{j}^{(N)}\right)$.

4. If the system $\Phi(n, N) \boldsymbol{\lambda}=\mathrm{c}$ has a nonnegative solution, accept the solution found, and finish.

5. If no nonnegative solution is found, set $i=i+1$, go to 2 .

Note the generality of the framework. We place no restrictions on the shape of $T$, other than compactness. It may or may not be convex, simply connected, arcwise connected, or discrete. The functions $\phi_{0}, \phi_{1}, \cdots, \phi_{n}$ are required to be continuous and linearly independent on $T$. They may be in several variables, be a Chebyshev system, or be a set of solutions to some partial differential equation. We can thus create at will formulas which are of "high precision" with respect to a particular class of basic functions. From a practical point of view, however, we are restricted in that we have to be able to precalculate $L \phi_{0}, L \phi_{1}, \cdots, L \phi_{n}$. Another restriction of course lies in the matrix size requirements of the particular linear programming code used.

We might, following Davis, take $T_{N}$ to be the first $N+1$ points of dense sequence in $T$. However, in practice, we have used sets $T_{N}$, such that $\bigcup_{N=1}^{\infty} T_{N}$ is dense in $T$. For example, on the interval $[-1,1]$, one might choose the roots of the Legendre polynomial $P_{N}$ for the set $T_{N}$.

\section{Examples.}

Example 1. Let us consider $\phi_{i}(t)=t^{i}, i=0,1, \cdots, n$, defined on $[-1,1]$, and let $I_{N} \equiv\{-1+2 i / N, i=0,1, \cdots, N\}$. Since we know the Cotes rules, we have that for $N=n, n=2,3, \cdots, 7,9, I_{N}$ supports a nonnegative quadrature formula for the usual integral, (weight function $\equiv 1$ ). However, for $n=8,10,11,12, \cdots$, there is no positive-term quadrature formula. Using the programming algorithm and the scheme above, we have calculated the function $N(n), n=1,2, \cdots, 29$, defined to be, for given $n$, the least $N$ such that $I_{N}$ supports a nonnegative quadrature 
of degree $n$. These values appear in Table (3.1). Table (3.2) gives the coefficients and points for $n=8, N=9$. See Wilson [8] for additional details. Note that the solution obtained is not symmetric. One can easily adjust the scheme to produce symmetric solutions by working on the interval $[0,1]$, and ignoring the odd functions $t, t^{3}, \cdots$.

Example 2. Consider the simplex in the plane, with vertices $(0,0),(0,1),(1,0)$. The dimension of the space of 2-dimensional polynomials of degree $k$ is

$$
N_{k}=\left(\begin{array}{c}
2+k \\
k
\end{array}\right) \text {. }
$$

We took two distinct partitions on $[0,1]$, a "closed" partition,

$$
P_{1} \equiv\{0,1 / N, 2 / N, \cdots, 1\}, \quad N=N_{k}-1,
$$

and an "open" partition

$$
P_{2}=\{1 / 2 N, 3 / 2 N, \cdots, 2 N-1 / 2 N\}, \quad N=N_{k}-1,
$$

and formed two point sets $T_{1}$ and $T_{2}$ by taking the points of $P_{1} \otimes P_{1}$ and $P_{2} \otimes P_{2}$ which lay in the simplex. For $k=1,2,3,4,5$, both meshes supported nonnegative quadrature rules. Some similar experiments were tried in higher dimensions. Although the rules found do not compare favorably with known rules for the $n$-simplex with regard to the number of function evaluations required, they do illustrate how rules may be found for arbitrarily shaped regions and arbitrary nonnegative weights. For high degree, and large dimension, this scheme suffers the serious drawback that the matrices $\Phi(n, N)$ are quite large. Further, round-off errors in the linear programming algorithm may become quite large.

TABLE 3.1

Values of $N(n)$

\begin{tabular}{lrrrrrrrrrrrrrrr}
\hline$n$ & 1 & 2 & 3 & 4 & 5 & 6 & 7 & 8 & 9 & 10 & 11 & 12 & 13 & 14 & 15 \\
$N$ & 1 & 2 & 3 & 4 & 5 & 6 & 7 & 9 & 9 & 13 & 13 & 17 & 17 & 22 & 22 \\
$n$ & 16 & 17 & 18 & 19 & 20 & 21 & 22 & 23 & 24 & 25 & 26 & 27 & 28 & 29 & \\
$N$ & 26 & 26 & 32 & 32 & 38 & 38 & 45 & 45 & 52 & 52 & 61 & 61 & 69 & 69 & \\
\hline
\end{tabular}

TABLE 3.2

Weights and Points for $n=8$

Based on $I_{9}$

\begin{tabular}{ccc}
\hline$i$ & $t_{i}$ & $c_{i}$ \\
0 & -1.000000 & 0.0644420 \\
1 & -0.777778 & 0.3453348 \\
2 & -0.555556 & 0.0482143 \\
3 & -0.333333 & 0.3755357 \\
4 & -0.111111 & 0.2133482 \\
5 & +0.111111 & 0.0445982 \\
6 & +0.333333 & 0.4880357 \\
7 & +0.777778 & 0.3573884 \\
8 & +1.000000 & 0.0631027 \\
\hline
\end{tabular}


TABLE 3.3

Some Point Sets Which Support

Nonnegative Quadrature Formula

\begin{tabular}{|c|c|c|c|c|c|}
\hline $\begin{array}{l}\text { base } \\
\text { functions }\end{array}$ & \multicolumn{5}{|c|}{$1, t,|t|$} \\
\hline $\begin{array}{l}\mathrm{Pt} \\
\text { Set }\end{array}$ & 2 & 3 & 4 & 5 & 6 \\
\hline $\begin{array}{l}G_{N} \\
L_{N} \\
C_{N}\end{array}$ & $\begin{array}{l}+ \\
+ \\
+\end{array}$ & $\begin{array}{l}+ \\
+ \\
+\end{array}$ & $\begin{array}{l}+ \\
+ \\
+\end{array}$ & $\begin{array}{l}+ \\
+ \\
+\end{array}$ & $\begin{array}{l}+ \\
+ \\
+\end{array}$ \\
\hline $\begin{array}{l}\text { base } \\
\text { functions }\end{array}$ & \multicolumn{5}{|c|}{$1, t,|t|, t^{2}, \sin (t), \exp (t)$} \\
\hline 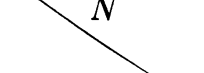 & 5 & 6 & 7 & 8 & 9 \\
\hline $\begin{array}{l}G_{N} \\
L_{N} \\
C_{N}\end{array}$ & $\begin{array}{l}\overline{-} \\
-\end{array}$ & $\begin{array}{l}+ \\
+ \\
+\end{array}$ & $\begin{array}{l}+ \\
+\end{array}$ & $\begin{array}{l}+ \\
+ \\
+\end{array}$ & $\begin{array}{l}+ \\
+ \\
+\end{array}$ \\
\hline $\begin{array}{l}\text { base } \\
\text { functions }\end{array}$ & \multicolumn{5}{|c|}{$1,|t|, t^{2},|t|^{3}$} \\
\hline & 5 & 6 & 7 & 8 & 9 \\
\hline $\begin{array}{l}G_{N} \\
L_{N} \\
C_{N}\end{array}$ & $\begin{array}{l}- \\
-\end{array}$ & $\begin{array}{l}+ \\
+\end{array}$ & $\frac{+}{-}$ & $\begin{array}{l}+ \\
+ \\
+\end{array}$ & $\begin{array}{l}+ \\
+ \\
+\end{array}$ \\
\hline $\begin{array}{l}\text { base } \\
\text { functions }\end{array}$ & \multicolumn{5}{|c|}{$1, t, t^{2}, t^{3},(t)_{+},\left(t+\frac{1}{2}\right)_{+}$} \\
\hline & 5 & 6 & 7 & 8 & 9 \\
\hline $\begin{array}{l}G_{N} \\
L_{N} \\
C_{N}\end{array}$ & $\begin{array}{l}\overline{+} \\
+\end{array}$ & $\frac{t}{+}$ & + & $\begin{array}{l}+ \\
+ \\
+\end{array}$ & $\begin{array}{l}+ \\
+ \\
+\end{array}$ \\
\hline
\end{tabular}

Example 3. Finally, experiments were carried out for some rather nonstandard base functions defined on $[-1,1]$, none of which are Chebyshev systems. However, it seemed reasonable to try the abscissas of some rather well-known positive-term integration rules, of polynomial precision $2 N+1,2 N-1$, and $N$, respectively. The point sets are

$$
\begin{aligned}
G_{N} & \equiv\{\text { abscissas in Gauss-Legendre } N+1 \text { point rule, }[5, \text { p. 100] }\} \\
L_{N} & \equiv\{\text { abscissas in Gauss-Lobatto } N+1 \text { point rule, }[5, \text { p. } 318]\} \\
C_{N} & \equiv\{\cos (i \pi / N), i=0,1, \cdots, N, \text { see }[3]\}
\end{aligned}
$$


In Table (3.3), we show, for the set of base functions indicated, those point sets which support a nonnegative quadrature formula (w.r.t. the base functions) by $a+$, and those which do not by $a-$. Note that the function $f(t)=(t)_{+}$is defined to be zero, if $t<0$, and $t$, for $t \geqq 0$.

The actual rules, which may be of some interest in their own right, are not of primary concern here. Rather, we have shown a general algorithmic procedure, computationally feasible, to obtain representations of strictly positive linear functionals on the spans of some rather unclassical sets of base functions which are not Chebyshev sets. Further examples are available upon application to the author.

4. Conclusion. In all the computed examples, the rule obtained was checked by using it to numerically integrate each base function, and comparing the result obtained with the known value supplied to the algorithm. In all cases, the results were accurate to at most the last three significant figures in the precision of arithmetic used. If round-off produces results which are not very accurate, one now has a set of base points which support a formula, and more accurate results can now be obtained, for example, by matrix inversion or iterative correction procedures.

This algorithm compares quite favorably with other algorithms which have been devised by the author. The problem of finding $T_{n}=\left\{t_{0}, t_{1}, \cdots, t_{n}\right\}$, such that the system (1.1) has a nonnegative solution, is essentially nonlinear. The approach described here is in effect a linearization. Thus, it seems reasonable, that except for extremely adverse functions and sets of base points, this algorithm is much more efficient and accurate than nonlinear algorithms, and this has been borne out by the author's experience.

The author should like to express his thanks to Professor P. J. Davis, who introduced the problem, and directed the author's thesis. The author should also like to thank the referee for his valuable comments.

IBM Research Center

Yorktown Heights, New York 10598

1. P. J. Davis, "A construction of nonnegative approximate quadratures," Math. Comp., v. 21, 1967, pp. 578-587. MR 36 \#5584.

2. P. J. DAvIs, "Approximate integration rules with nonnegative weights," in Lecture Series in Differential Equations, Georgetown University, Washington, D. C., 1967.

3. W. Fraser \& M. W. Wilson, "Remarks on the Clenshaw-Curtis quadrature scheme," SIAM Rev., v. 8, 1966, pp. 322-327. MR 34 \#3784.

4. W. W. RogosinskI, "On non-negative polynomials," Ann. Univ. Sci. Budapest, Eötvös Sect. Math., v. 3-4, 1961, pp. 253-280. MR 26 \#3843.

5. A. H. STroud \& D. SECrest, Gaussian Quadrature Formula, Prentice-Hall, Englewood Cliffs, N. J., 1966. MR 34 \#2185.

6. V. TCHAKALOFF, "Formules de cubatures mécaniques à coefficients non-négatifs," Bull. Sci. Math., (2) v. 81, 1957, pp. 123-134. MR 20 \#1145.

7. M. W. WILson, Geometric Aspects of Quadratures with Non-Negative Weights, Ph.D. Thesis, Brown University, 1968.

8. M. W. WILSON, Approximation of Non-Negative Continuous Linear Functionals, Brown University Technical Report, May, 1968. 